\title{
A Survey on Migraine Prevalence in Patients with Inflammatory Bowel Disease - A Single Centre Experience
}

\author{
Somaye Chehel Cheraghi ${ }^{1 *}$, Nasser Ebrahimi Daryani ${ }^{2}$, Mojdeh Ghabaee ${ }^{3}$
}

\begin{abstract}
(n)
1. Internal Medicine Resident, Gastroenterology Department, Tehran University of Medical Sciences, Tehran, Iran

2. Professor, Gastroenterology Department, Tehran University of Medical Sciences, Tehran, Iran

3. Associate Professor, Neurology Department, Tehran University of Medical Sciences, Tehran, Iran
\end{abstract}

\footnotetext{
* Corresponding Author:

Somaye Chehel Cheraghi, MD Gastroenterology Department, Imam Khomeini Hospital, Tehran University of Medical Sciences, Tehran, Iran Tel: + 9821666939010 Fax: + 982166581615 Email: s.40cheragh@yahoo.com
}

Received: 06 Jun. 2016 Accepted: 17 Sep. 2016

\section{ABSTRACT}

\section{BACKGROUND}

It is hypothesized that migraine may be related to inflammatory bowel disease (IBD), therefore in this cross-sectional study we evaluated the prevalence of migraine in patients with IBD.

\section{METHODS}

In this cross-sectional study 80 patients with IBD and 80 patients without IBD referring to a private gastroenterology clinic from May to January 2014 were evaluated regarding the prevalence of migraine, severity of migraine based on Headache Impact Test (HIT-6), and habits related to headache.

\section{RESULTS}

160 participants with the mean age of 35 years were evaluated. The prevalence of migraine in the case group was significantly higher than the control $(21.3 \%$ vs. $8.8 \%, p=0.027)$. Moreover, duration of each attack (hours) in IBD group was significantly higher than the control group $(p<0.001)$ while the duration of migraine involvement (months) and number of attacks was higher in the control group ( $p=0.019$ and 0.048 , respectively). Headache other than migraine in the control group was significantly higher than the IBD group $(p<0.001)$. Disability in the case group was more than the control group but the difference was not significant. The correlation between the severity of disability related to migraine (based on HIT-6) and severity of IBD (based on Mayo score \& Crohn's disease activity index (CDAI)) was not significant $(\mathrm{r}=0.16$, $p=0.58$ ). Moreover the correlation between the duration of IBD and migraine prevalence was not significant $(\mathrm{r}=-0.14, p=0.19)$.

\section{CONCLUSION}

We found that the prevalence of migraine in patients with IBD is significantly more than normal population. More studies are needed to highlight the correlation between migraine and IBD.

\section{KEYWORDS}

Migraine, Inflammatory Bowel Disease, Headache, HIT-6, CDAI.

Please cite this paper as:

Chehel Cheraghi S, Ebrahimi Daryani N, Mojdeh Ghabaee. A survey on migraine prevalence in patients with inflammatory bowel disease - a single centre experience. Middle East J Dig Dis 2016;8:282-288. DOI: 10.15171/mejdd.2016.37

\section{INTRODUCTION}

Inflammatory bowel disease (IBD) is a complex chronic inflammatory disorder including ulcerative colitis (UC) and Crohn's disease (CD), which affects about $0.4 \%$ of general population in developed countries. ${ }^{1}$ It is well established that IBD is associated with several important extraintestinal manifestations and in some patients they are the first clinical manifestations of IBD. The incidence of neurological manifestations of IBD is not clear but in some 
reports it is estimated about 0.25 to $37.5 \% .^{2-5}$

There are several evidence showing that IBD affects the central nervous system. ${ }^{6-8}$ Migraine is a neurological disease with unknown origin, however a genetic background and some environmental factors play an important role in its pathophysiology. ${ }^{9-10}$

Studies about the correlation between IBD and migraine are rare, however Ford and colleagues in a retrospective study included 100 patients with IBD and revealed migraine manifestation in $30 \%$ of the patients. They emphasized that the prevalence of migraine in the United States was about $18.2 \%$ for women and $6.5 \%$ for men. ${ }^{11}$ In line with that study another report by Dimitrova also showed a higher rate of migraine among patients with IBD than controls. ${ }^{12}$ The exact cause of this correlation is not fully understood, however migraine and IBD are related to systemic endothelial dysfunction and inflammation. ${ }^{13,14}$

Furthermore, other studies indicated that migraine might occur as a side effect of immunosuppressive treatment in patients with IBD. ${ }^{15}$ However the correlation is not linear and there are not any data showing that any improvement in IBD will lead to improvement of migraine symptoms in patients with IBD. ${ }^{16}$ So to address these concerns and to estimate the prevalence of migraine in patients with IBD we performed this crosssectional study and investigated this correlation.

\section{MATERIALS AND METHODS}

In this cross-sectional study 80 patients with IBD (CD \& UC) referring to a private gastroenterology clinic from May to January 2014 were enrolled as the case group. Moreover after adjustment for sex and age, 80 participants without IBD were selected from the family members of the patients as a control group.

The study protocol was explained for all the participants and written informed consent was taken. Criteria for enrolment were age more than 18 years, having IBD, and signed consent. On the other hand the patients were excluded if they had history of head trauma, vascular complications, surgery on head and neck, brain tumor and lumbar puncture in last 3 years or at uncertain time.

IBD was diagnosed based on clinical symptoms, laboratory findings, endoscopy, colonoscopy, biopsy, imaging studies, pathological findings and exclusion of other etiologies. The severity of Crohn's disease was assessed based on Crohn's disease activity index (CDAI) and severity of ulcerative colitis according to Mayo score. ${ }^{17-18}$

Age, sex, history of anxiety and depression, family history of inflammatory bowel disease and migraine headache, the usage history of any medications particularly Selective serotonin reuptake inhibitors (SSRIs), opioids, Nonsteroidal anti-inflammatory drugs (NSAIDs), Tricyclic antidepressants (TCAs), Oral contraceptive pills (OCP) and Hormone replacement therapy (HRT) were recorded. Additionally, history of smoking, alcohol usage, coffee consumption and any drug abuse were recorded for all the participants.

All the patients were asked about the frequency of headache with duration of 4-72 hours and according to Headache International Society (HIS) criteria, migraine was diagnosed. Migraine was diagnosed if there were two of the four criteria including unilateral pain, pulsatile pain, exacerbation of headache by movement, and moderate to severe headache, and at least one criteria of the two criteria including nausea, vomiting and photophobia or phonophobia. If migraine was diagnosed for a patient, then the duration of pain, frequency, initiation of headache before or after IBD, triggers of headache, and improvement of headache after treatment of IBD would be recorded. The level of disability related to migraine was assessed based on Headache Impact Test-6 (HIT-6) HIT-6 is a well-recognized tool based on a questionnaire to measure the headache impact on patient's ability to function in normal daily life. The score is classified in different levels from 49 or less (little or no impact), 5055 (some impact), 56-59 (substantial impact) and 60 or more (very severe impact), each of which needs specific management ${ }^{19}$. Patients who did not meet the migraine criteria were categorized as patients with headache other than migraine.

\section{Statistical analysis}

Data were analyzed using SPSS software version 16. Categorical data are presented as numbers (\%), and continuous data as mean $\pm \mathrm{SD}$. We used Chi square or Fisher's exact test to compare categorical variables and Mann-Whitney's rank sum U test to compare continuous variables. Correlations between variables were calculated by Pearson or Spearman rank correlation analysis. $p<0.05$ 
was considered as statistically significant.

\section{RESULTS}

In this cross-sectional study 160 participants (80 with IBD as case group and 80 without IBD as control group) with mean age of 35 years were evaluated.

The difference between the two groups regarding sex and age was not significant. The prevalence of migraine in the case group was significantly higher than the control group $(21.3 \%$ vs. $8.8 \%, p=0.027)$ (table 1$)$. Moreover, the duration of each attack (hours) in IBD group was higher than the control group $(p<0.001)$ while the duration of migraine involvement (months) and number of attacks were significantly higher in the control group ( $p=0.019$ and $p=0.048$, respectively, table 2 and 3). Additionally, headache other than migraine in the control group was significantly higher than the IBD group ( $p$ $<0.001$, table1). Disability in the case group was more than the control group but the difference was not significant (table3). The correlation between the severity of disability related to migraine (based on HIT-6) and severity of IBD (based on Mayo score \& CDAI) was not significant $(\mathrm{r}=0.16, p=0.58)$. Moreover correlation between the duration of IBD and migraine prevalence was not significant $(\mathrm{r}=-0.14, p=0.19)$. Migraine occurred in $10(58.8 \%)$ patients before IBD and in $7(41.2 \%)$ patients after IBD $(p=0.043)$. NSAID consumption was significantly higher in control group $(p=0.005)$ while corticosteroid, 5-aminosalisilic acid (5-ASA) and azathioprine were significantly more used in IBD group $(p<0.001)$. There was not any important difference between two groups about SSRI, TCA, OCP and HRT usage ( $p=0.35$, $p=0.21, p=0.995$ respectively), (Table 1 ).

\section{DISCUSSION}

Neurologic manifestations of inflammatory bowel disease (IBD) is a major health problem and may affect peripheral and central nervous system. ${ }^{10-15}$ In this cross-sectional study we found that the frequency of migraine in the IBD group was significantly higher than the control group (21.3\% vs. 8.8\%, $p=0.027$ ). Moreover, the duration of each attack (hours) was significantly higher in the IBD group while the duration of migraine involvement (months) and the frequency of attacks were higher in the control group. A study by Oliveira and colleagues in 2008 supported our results and reported migraine in $25 \%$ of patients with IBD. Moreover the researchers emphasized that headache occurred in $55.3 \%$ of the patients with IBD. ${ }^{17}$ In line with our findings a study by Dimitrova and co-workers in 2013 revealed that the frequency of migraine in patients with IBD was significantly more than controls (14\% vs. $6 \%$, $p=0.02$ ), and chronic headache in patients with IBD was significantly more than controls ( $23 \%$ vs $14 \%, p<0.001) .{ }^{12}$ Nevertheless, the question still remains; why the prevalence of migraine in patients with IBD is higher than normal population? In this regard some studies concluded that abdominal complaints may play an important role in pathophysiology of migraine in such patients. These studies confirmed that abdominal complaints have been related to headache, depression, somatisation, and conversion disorders. ${ }^{18-19}$ In Keeping with this hypothesis, in the current practice the frequency of anxiety in patients with IBD was significantly more than the control group. Moreover several studies revealed the role of inflammation in migraine. These studies indicated C-reactive protein (CRP), Matrix metallopeptidase 9 (MMP-9), cytokines, adhesion molecules, Nuclear factor kappa-light-chain-enhancer of activated B cells (NF-kB), and Inducible nitric oxide synthase (iNOS) have been involved in migraine. ${ }^{20-23}$ In the present experience consumption of SSRIs, TCA, OCP/ HRT in the case and control groups did not differ significantly, however the difference between the two groups regarding NSAID, corticosteroid, 5-ASA, and azathioprine intake was significant. In line with our findings, previous practices reported no correlation between migraine and OCP/HRT ${ }^{24-26}$, however in contrast to our findings some authors revealed no correlation between migraine and NSAIDs. ${ }^{27-28}$ Higher NSAID consumption in the control group may be attributed to its lower prescription in the case group due to the concern about its role in IBD exacerbation, although this is just a hypothesis and its relationship needs to be studied in future. In parallel with a significant difference between two groups in terms of specific foods intake (such as restriction of dairy products in case group) $(p<0.001)$, a statistically significant difference between the two groups regarding the regimen as a migraine headache trigger was also found $(p=0.039)$. Therefore, regimen seems to be more impressive in IBD group. Similarly, some authors demonstrated a remarkable improvement in headache severity after initiation of 
Table 1: Patients' characteristics in the two groups

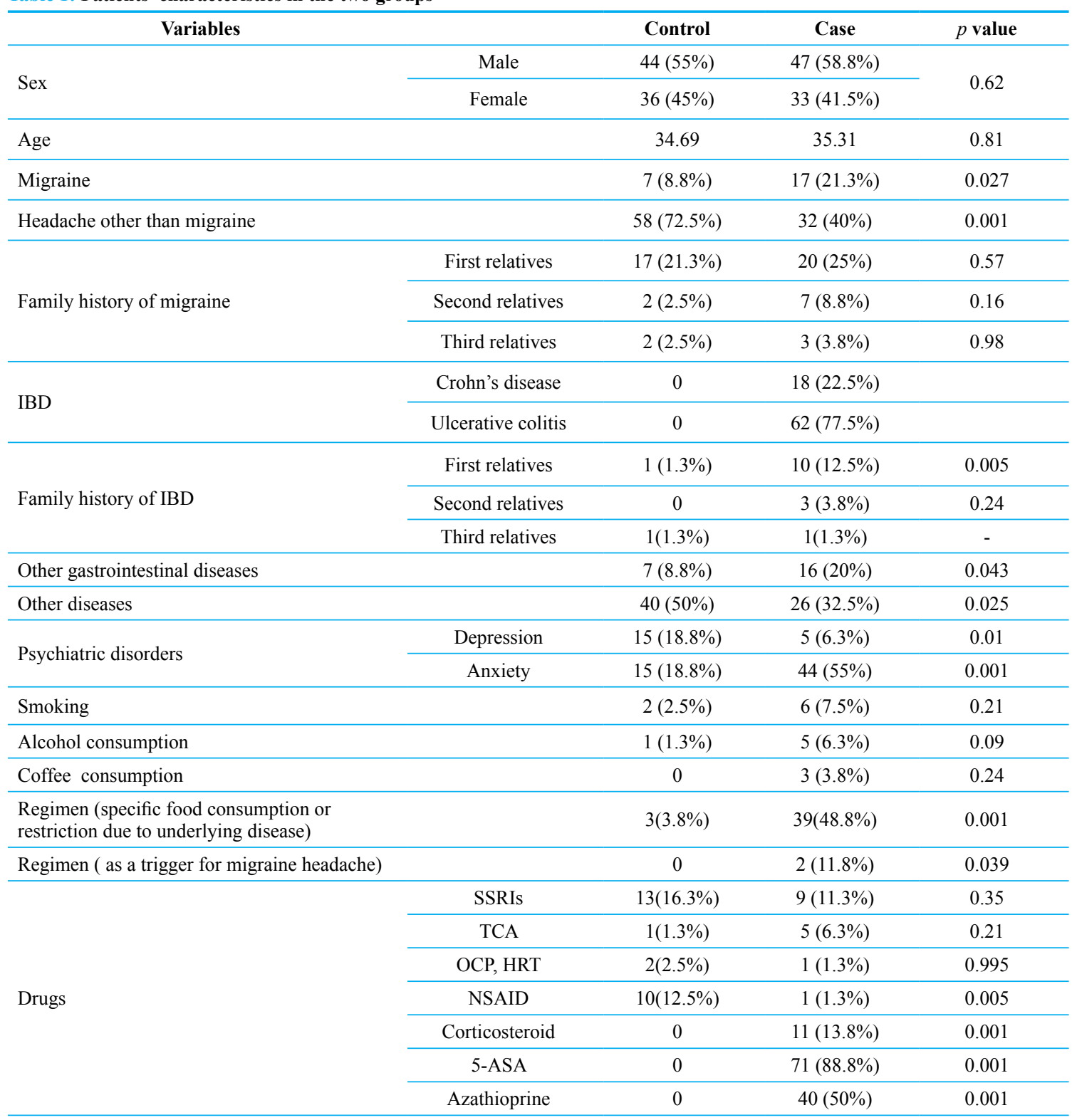

specific regimen for patients with IBD. ${ }^{29-30}$ In the present study, the severity of IBD and migraine did not show a significant correlation that is in agreement with the study by Dimitrova and colleagues. ${ }^{12}$

In summary our practice was in accord with a few studies in this field, however there are a number of limitations to this study that warrant mention such as relatively small sample size and no follow up. More studies with larger series and in a cohort study are required to validate results reported here.
Conclusion: We revealed that the frequency of migraine in patients with IBD is significantly more than the normal population. More studies are needed to highlight the correlation between migraine and IBD.

\section{ACKNOWLEDGEMENTS}

We would like to thank the administrative, and secretarial staff of the Dr Daryani's office for their contribution to the maintenance of our patients' re- 
Table 2: The characteristics of headache in the patients of the two groups

\begin{tabular}{|c|c|c|c|c|}
\hline Variables & & Control & Case & $p$ value \\
\hline \multirow{5}{*}{ Number of attacks (per week) } & 0.5 & 0 & $3(17.6 \%)$ & \multirow{5}{*}{0.048} \\
\hline & 1 & 0 & $6(35.3 \%)$ & \\
\hline & 2 & $2(28.6 \%)$ & $5(29.4 \%)$ & \\
\hline & 3 & $2(28.6 \%)$ & $1(5.9 \%)$ & \\
\hline & 4 & $3(42.9 \%)$ & $2(11.8 \%)$ & \\
\hline \multirow{9}{*}{ Duration of attacks (hour) } & $1-6$ & $6(85.7 \%)$ & $3(17.6 \%)$ & \multirow{9}{*}{0.001} \\
\hline & $6-12$ & $1(14.3 \%)$ & $1(5.9 \%)$ & \\
\hline & $12-18$ & 0 & $2(11.8 \%)$ & \\
\hline & $18-24$ & 0 & $3(17.6 \%)$ & \\
\hline & $24-30$ & 0 & $3(17.6 \%)$ & \\
\hline & $36-42$ & 0 & $1(5.9 \%)$ & \\
\hline & $42-48$ & 0 & $2(11.8 \%)$ & \\
\hline & $48-54$ & 0 & $1(5.9 \%)$ & \\
\hline & $>72$ & 0 & $1(5.9 \%)$ & \\
\hline \multirow{5}{*}{ Migraine triggers } & Menstruation & $1(14.3 \%)$ & $4(23.5 \%)$ & 0.071 \\
\hline & Insomnia & $4(57 \%)$ & $9(52.9 \%)$ & 0.079 \\
\hline & Regimen & 0 & $2(11.8 \%)$ & 0.039 \\
\hline & Starvation & $3(42.9 \%)$ & $10(58.8 \%)$ & 0.65 \\
\hline & Stress & $3(42.9 \%)$ & $10(58.8 \%)$ & 0.65 \\
\hline
\end{tabular}

Table 3: The frequency of disability related to migraine and duration of headache disease

\begin{tabular}{|c|c|c|c|c|}
\hline Variables & & Control & Case & $p$ value \\
\hline \multirow{4}{*}{ Disability (HIT-6 Score) } & $\leq 49$ & $1(14.3 \%)$ & $4(23.5 \%)$ & 0.10 \\
\hline & $50-55$ & $1(14.3 \%)$ & 0 & 0.29 \\
\hline & $56-59$ & $3(42.9 \%)$ & $2(11.8 \%)$ & 0.12 \\
\hline & $\geq 60$ & $2(28.6 \%)$ & $11(64.7 \%)$ & 0.18 \\
\hline \multirow{13}{*}{$\begin{array}{l}\text { Duration of migraine } \\
\text { involvement (months) }\end{array}$} & $12-24$ & 0 & $2(8.11 \%)$ & \multirow{13}{*}{0.019} \\
\hline & $24-36$ & 0 & $4(5.23 \%)$ & \\
\hline & $36-48$ & 0 & $1(5.9 \%)$ & \\
\hline & $48-60$ & 0 & $1(5.9 \%)$ & \\
\hline & $60-72$ & 0 & $2(8.11 \%)$ & \\
\hline & $72-84$ & 0 & $1(5.9 \%)$ & \\
\hline & $84-96$ & 0 & $1(5.9 \%)$ & \\
\hline & $96-108$ & $1(14.3 \%)$ & $1(5.9 \%)$ & \\
\hline & $108-120$ & $1(14.3 \%)$ & $1(5.9 \%)$ & \\
\hline & $120-132$ & $4(57.1 \%)$ & 0 & \\
\hline & $132-144$ & $1(14.3 \%)$ & 0 & \\
\hline & $144-156$ & 0 & $1(5.9 \%)$ & \\
\hline & $168-180$ & 0 & $2(8.11 \%)$ & \\
\hline
\end{tabular}


cords without which this project would have been impossible.

\section{CONFLICT OF INTEREST}

The authors declare no conflict of interest related to this work.

\section{REFERENCES}

1. Watts DA, Satsangi J. The genetic jigsaw of inflammatory bowel disease. Gut 2002;50: III 31-6. doi: 10.1136/ gut.50.suppl_3.iii31.

2. Elsehety A, Bertorini TE. Neurologic and neuropsychiatric complications of Crohn's disease. South Med J 1997;90:60610. doi: 10.1097/00007611-199706000-00005

3. Greenstein AJ, Janowitz HD, Sachar DB. The extra-intestinal complications of Crohn's disease and ulcerative colitis: a study of 700 patients. Medicine (Baltimore) 1976;55:40112. doi:10.1097/00005792-197609000-00004

4. Rankin GB, Watts HD, Melnyk CS, Kelley ML Jr. National Cooperative Crohn's Disease Study: extraintestinal manifestations and perianal complications. Gastroenterology 1979;77:914-20.

5. Lossos A, River Y, Eliakim A, Steiner I. Neurologic aspects in inflammatory bowel disease. Neurology 1995;45:41621. doi: 10.1212/WNL.45.3.416

6. Konturek SJ, Konturek JW, Pawlik T, Brzozowski T. Brain-gut axis and its role in the control of food intake. $J$ Physiol Pharmacol 2004;55:137-54.

7. Derbyshire SWG. A systematic review of neuroimaging data during visceral stimulation. Am J Gastroenterol 2003;98:12-20. doi:10.1111/j.1572-0241

8. Scheid R, Teich N. Neurologic manifestations of ulcerative colitis. Eur J Neurol 2007;14:483-93. doi: 10.1111/ j.1468-1331.

9. RussellM, OlesenJ. Increased familial risk and evidence of genetic factor in migraine. BMJ 1995 311:541-4. doi:10.1136/bmj.311.7004.541

10. BelkaidY, NaikS. Compartmentalized and systemic control of tissue immunity by commensals. Nat Immunol 2013;14:646-53. doi:10.1038/ni.2604

11. Ford S, Finkel AG, Isaacs KL. Migraineinpatientswithinflammatory boweldisorders. $J$ Clin Gastroenterol 2009;43:499. doi:10.1097/MCG. 0b013e318188be85

12. Dimitrova AK, Ungaro RC, Lebwohl $\mathrm{B}$, Lewis SK,Tennyson CA,Green MW, et al. Prevalence of migraine in patients with celiac disease and inflammatory bowel disease. Headache 2013;53:344-55. doi:10.1111/j.1526-4610.2012. 02260.x

13. Vanmolkot FH, Van Bortel LM, de Hoon JN. Altered arterial function in migraine of recent onset. Neurology 2007;68:156370. doi: 10.1212/01.wnl.0000260964.28393.ed

14. Chidlow JH Jr, Shukla D, Grisham MB, Kevil CG.
Pathogenic angiogenesis in IBD and experimental colitis: new ideas and therapeutic avenues. Am J Physiol Gastrointest Liver Physiol 2007;293:G5-G18. doi: 10.1152/ ajpgi.00107.2007

15. Fellermann K, Steffen M, Stein J, Raedler A, Hämling J, Ludwig D, et al. Mycophenolate mofetil: lack of efficacy in chronic active inflammatory bowel disease. Aliment Pharmacol Ther 2000;14:171-6. doi: 10.1046/j.1365-2036.2000.00695.x

16. van Hemert S, Breedveld AC, Rovers JMP, Vermeiden JPW, Witteman BJM, Smits MG, et al. Migraine associated with gastrointestinal disorders: review of the literature and clinical implications . Front Neurol 2014;5:2418. doi: 10.3389/fneur.2014.00241.

17. Oliveira GR, Teles BC, Brasil EF, Souza MH, Furtado LE, de Castro-Costa CM, et al. Peripheral neuropathy and neurological disorders in an unselected Brazilian population-based cohort of IBD patients. Inflam Bowel Dis 2008;14:389-395. doi:10.1002/ibd.20304

18. Hillila MT, Hamalainen J, Heikkinen ME, Farkkila MA. Gastrointestinal complaints among subjects with depressive symptoms in the general population. Aliment Pharmacol Ther 2008;28:648-54. doi:10.1111/j.13652036.2008.03771.x

19. Howell S, Poulton R, Caspi A, Talley NJ. Relationship between abdominal pain subgroups in the community and psychiatric diagnosis and personality.A birth cohort study. J Psychosom Res 2003;55:179-87. doi:10.1016/ S0022-3999(02)00599-8

20. Sarchielli P, Floridi A, Mancini ML, Rossi C, Coppola F, Baldi A, et al. NF-kappaB activity and iNOS expression in monocytes from internal jugular blood of migrainewithout aura patients during attacks. Cephalalgia 2006;26:10719. doi: 10.1111/j.1468-2982.2006.01164.x

21. Sarchielli P, Alberti A, Baldi A, Coppola F, Rossi C, Pierguidi L, et al. Proinflammatory cytokines, adhesion molecules, and lymphocyte integrin expression in the internal jugular blood of migraine patients without aura assessed ictally. Headache 2006;46:200-7. doi: 10.1111/j.15264610.2006.00337.x

22. Leira R, Sobrino T, Rodriguez-Yanez M, Blanco M, Arias S, Castillo J. Mmp-9 immunoreactivity in acute migraine. Headache 2007;47:698-702. doi: 10.1111/j.15264610.2006.00641.x

23. Vanmolkot FH, de Hoon JN. Increased C-reactive protein in young adult patients with migraine. Cephalalgia 2007;27:843-6. doi: 10.1111/j.1468-2982.2007.01324.x

24. Allais G, Gabellari IC, Airola G, Borgogno P, Schiapparelli P, Benedetto C. Headache induced by the use of combined oral contraceptives. Neurol Sci 2009;30:S15-S17. doi: 10.1007/s10072-009-0051-9

25. Machado RB, Pereira AP, Coelho GP, Neri L, Martins L, Luminoso D. Epidemiological and clinical aspects of migraine in users of combined oral contraceptives. Contraception 2010;81:202-8. doi:10.1016/j.contraception.2009.09.006 
26. Aegidius KL, Zwart JA, Hagen K, Schei B, Stovner LJ. Hormone replacement therapy and headache prevalence in postmenopausal women. The Head- HUNT study. Eur J Neurol 2007;14:73-8. doi: 10.1111/j.14681331.2006.01557.x

27. Cupini LM, Sarchielli P, Calabresi P. Medication overuse headache: Neurobiological, behavioural and therapeutic aspects. Pain 2010;150:222-4. doi: 10.1016/j.pain.2010.05.003

28. Leone M, Attanasio A, Croci D, Filippini G, D'A mico D, Grazzi L, et al. The serotonergic agent m-chlorophenylpiperazine induces migraine attacks: A controlled study. Neurology 2000;55:136-9. doi: 10.1212/WNL.55.1.136.

29. Serratrice J, Disdier P, de Roux C, Christides C, Weiller PJ. Migraine and coeliac disease. Headache 1998;38:6278. doi:10.1046/j.1526-4610.1998.3808627.x

30. Gabrielli M, Cremonini F, Fiore G, Addolorato G, Padalino C, Candelli M, et al. Association between migraine and celiac disease: Results from a preliminary case-control and therapeutic study. Am J Gastroenterol 2003;98:625-9. doi:10.1111/j.1572-0241.2003.07300.x 